\title{
Prevention and management of multimorbidity to ensure healthcare sustainability
}

Kelvin Bryan Tan ${ }^{1,2,3}$ PhD

Singapore has a healthcare system that is distinguished by its ability to achieve top health outcomes at very low healthcare expenditures. ${ }^{1}$ Yet one of the Ministry of Health's (MOH) foremost policy concerns is the sustainability of the healthcare system in the face of a rapidly ageing society. With an ageing demographics, Singapore's old-age support ratio will decrease from 4.8 in 2018 to 2.7 in $2030 .^{2}$ This falling old-age support ratio implies that financing of healthcare expenditures will double because these expenses are largely concentrated on the population above 65 years old.

The stark reality of an ageing demographics necessitates a strong need to identify measures that can reduce future healthcare burden. This burden will not just be in the increased healthcare expenditures, but also the medical, nursing and allied health manpower that will be required to treat and take care of a rapidly ageing population.

One important way to reduce future healthcare burden is to improve the management of chronic conditions and those with multimorbidities. Research by Tan et al. published in this issue of the Annals fills an important and critical gap in ascertaining the additional expenditures of those with multimorbidities. ${ }^{3}$ While there are many available studies in other jurisdictions, there are few publications to date in Singapore. This study finds that multimorbidity doubles primary healthcare costs-from SGD318 to SGD695, with an increment of SGD125 to SGD200 annual costs per additional comorbidity beyond the second. The analysis of difference in annual costs between patients with multimorbidity and those without is very consistent across sex, ethnicity and age groups, and these differences are much larger than those across patient characteristics. This implies that the number of morbidities is a more important determinant of healthcare expenditure than age.

Clearly these differences in expenditures are attributable to the more intensive treatment and more regular follow-ups required to treat those with multimorbidities. It is for these reasons that $\mathrm{MOH}$ has tiered financing schemes. Starting 1 January 2021, patients with complex chronic conditions can claim up to SGD700 of MediSave - the national medical savings scheme-for treatment under the Chronic Disease Management Programme as compared to SGD500 for other chronic conditions. The Community Health Assist Scheme (CHAS) subsidies are also similarly tiered with general practitioners (GPs) receiving higher government subsidies for managing patients with more complex chronic conditions.

Given these higher expenses, one of the ways to reduce future healthcare expenditures is secondary prevention of multimorbidities, such as better management of hypertension to prevent stroke or kidney disease. Studies have shown that screening and optimal management of these chronic conditions can be highly cost effective. ${ }^{4}$

Tan et al. identified the top 10 triads by prevalence with "diabetes, hypertension and hyperlidaemia" topping the list, and subsequent triads involving at least 2 of these conditions. It is thus unsurprising that in 2016, MOH declared a War on Diabetes to drive collective action by patients, families, communities and providers to reduce the burden of diabetes in our population..$^{5}$ Among these initiatives, one of the most critical is the Holistic Approach in Lowering and Tracking Chronic Kidney Disease (HALT-CKD) Programme implemented in 2017 in all public primary care clinics in Singapore. Early findings from this programme have shown some promising results in slowing the progression of chronic kidney disease. ${ }^{6}$

It is important to reiterate that it is the multimorbid conditions that should be averted, and not the costs associated with the management of these conditions. These primary care costs are "good" costs, which can potentially reduce future downstream expenses and loss in quality adjusted life years by averting complications

\footnotetext{
${ }^{1}$ Future Systems Office, Infocomm Technology and Data Group, Ministry of Health, Singapore

${ }^{2}$ Saw Swee Hock School of Public Health, National University of Singapore, Singapore

${ }^{3}$ Centre of Regulatory Excellence, Duke-NUS Medical School, Singapore

Correspondence: Dr Kelvin Bryan Tan, Future Systems Office, Infocomm Technology and Data Group, Ministry of Health, 16 College Rd, College of Medicine Building, Singapore 169854.

Email: Kelvin_Bryan_TAN@moh.gov.sg
} 
due to these chronic diseases. One limitation of this study is that we cannot ascertain whether these disease management costs reflect optimal management of patients, or whether there is underutilisation of care for some patients. If it is possible to follow up with these cohort of patients, it would be extremely useful to examine whether patients with better treatment adherence have lower costs and better outcomes over the long run, albeit with higher disease management costs in the short term.

We hope this study drives greater investment in chronic disease management programmes, as well as population health initiatives. Use of the estimates of additional costs of comorbidities, provided by the authors to derive cost-effectiveness analytical models, ought to establish that secondary prevention efforts by primary care providers to prevent or delay the onset of comorbidities are highly cost-effective if not cost-saving. These secondary preventive measures include regular diabetes screening for patients with hyperlipidaemia or hypertension, medication adherence to prevent the onset of stroke and progression of chronic kidney disease, among others.

It is important to highlight that most, if not all, of these measures are most appropriately done at the primary care level. Successful execution of these measures requires our primary care providers to be equipped with the necessary data flow and IT systems to track and monitor their patients with chronic diseases. Currently in Singapore, most of these efforts are often manually tracked and entirely reliant on the diligence and persistence of primary care doctors or clinic assistants to remind patients. Moving ahead, we need to rely on ITenabled solutions that can remind and nudge patients for better adherence to chronic disease management so that the time of our physicians and clinic assistants can be better spent. Adoption of telehealth and telemedicine can also further improve outcomes as well as optimise the use of scarce resources. Primary care providers should also be rewarded for their efforts to better manage patients with chronic conditions via an appropriate set of incentives.
The study also highlights the importance of primary prevention. Avoidance or even delay of chronic conditions via lifestyle changes reduces the resources required to manage these chronic conditions. Given the high prevalence of these chronic conditions, 30\% of Singapore's 5.45 million population requiring at least SGD300 would translate to a national healthcare expenditure of more than SGD500 million per year. To provide context, the government disbursed SGD180 million in CHAS subsidies to private GPs in fiscal year 2019.

Such primary and secondary preventive measures can only be undertaken by transforming primary care within a population health strategy. We have examples of Singapore's regional healthcare clusters moving towards empanelment, patient activation, and shifting away from a doctor-centric towards team-oriented delivery of care. ${ }^{7}$ More needs to be done.

\section{REFERENCES}

1. Tan KB, Tan WS, Bilger M, et al. Monitoring and Evaluating Progress towards Universal Health Coverage in Singapore. PLoS Med 2014;11:e1001695.

2. Ministry of Health Singapore. MOH Leads Review to Strengthen Caregiver Support for Seniors, 23 October 2018. Available at: https://www.moh.gov.sg/news-highlights/details/moh-leads-review-tostrengthen-caregiver-support-for-seniors. Accessed on 11 November 2021.

3. Tan SY, Lew KJ, Ying X, et al. Healthcare cost of patients with multiple chronic diseases in Singapore public primary care setting. Ann Acad Med Singap 2021;50:809-17.

4. Howard K, White S, Salkeld G, et al. Cost-Effectiveness of Screening and Optimal Management for Diabetes, Hypertension, and Chronic Kidney Disease: A Modeled Analysis. Value Health 2010;13:196-208.

5. Ow Yong L, Koe LWP. War on Diabetes in Singapore: a policy analysis. Health Res Policy Syst 2021;19:15.

6. Chua YT, Ngoh CLY, Teo BW. Making Sense of Chronic Kidney Disease in Primary Care - Identification, Evaluation and Monitoring. Singapore Fam Physician 2021;47:58-63.

7. Chong PN, Tang WE. Transforming primary care-the way forward with the TEAMS2 approach. Fam Pract 2019;36:369-70. 